\title{
Analysis of Gated Flux From or Into Sealed Membrane Fragments
}

\author{
J. Bernhardt and E. Neumann \\ Max-Planck-Institut für Biochemie, D-8033 Martinsried bei \\ München, Federal Republic of Germany \\ (Received 25 February 1980)
}

\begin{abstract}
Physico-chemical factors that determine tracer substance flux from or into sealed vesicular structures are examined. Flux amplitudes are dependent on the average volume of a vesicle, while flux rates depend on the average number of transmembrane channels per vesicle. Gating processes leading to channel opening and/or closing affect both amplitudes and rates. Averaging over inhomogeneities in vesicle size and channel density leads to an cxplicit expression for time-dependent tracer content. Means for experimentally determining all variable factors in this expression are discussed.
\end{abstract}

\section{Introduction}

Electrical excitability of biological cells involves the flow of ions through transmembrane channels. There is good evidence that the time course of excitation events is controlled by specialized gating molecules. Several electrical signals resulting from various types of gating processes are known: (a) action potentials of nerves and muscles; (b) rapid post-synaptic potential changes elicited by neuroactivator binding to acetylcholine receptors of fish electric organs and skeletal muscle; (c) slow post-synaptic potential changes thought to be mediated by cyclic nucleotides.

Biochemical characterization of the molecular apparatus underlying excitability involves isolation and purification of gating as well as ion transport components, though these need not be separate molecular entities. Unequivocal verification of the intactness of the purified components requires their reconstitution into a membrane, whereupon both gating and ion transport properties of the reassembled excitation apparatus must be determined. Measurement of the kinetics of tracer ion flux from or into sealed membrane fragments (microsacs) can be used to investigate gating events. Such ion flux studies have been performed using homogenates of fish electric organs (Kasai \& Changeux, 1971; Popot, Sugiyama \& Changeux, 1976; Hess et al., 1975; Bernhardt \& Neumann, 1978; Miller et al., 1978), 
vesicles containing reconstituted nerve sodium channels (Villegas et al., 1977, 1979), rat parotid acinar cells (Landis \& Putney, jr., 1979). At present, experimental techniques permitting resolution of flux events in the millisecond time range are being developed (Hess, Derek \& Aoshima, 1979).

In a previous publication (Bernhardt \& Neumann, 1978), a scheme for analysing flux kinetics was presented. For a well characterized example it was shown that (i) flux contribution controlled by a gating molecule can be determined from overall flux curves; (ii) both the time course and the amplitudes of ion flux reflect the gating process; (iii) physico-chemically well-founded parameters for gating events can be determined from flux data; (iv) inhomogeneities in size and composition of the membrane fragments can significantly distort ideal flux behaviour.

However, the treatment was incomplete in several respects. The number of activatable channels per microsac was represented by a continuously varying density. Channel opening following ligand-induced activation was expressed in terms of a fixed fraction of open channels per microsac. Actually a statistical distribution of the number of open channels is to be expected. Finally, it was not indicated how inhomogeneities in the preparation could be accounted and corrected for.

In this article a general expression linking gating kinetics to flux kinetics will be presented. The previously proposed scheme of analysis (Bernhardt $\&$ Neumann, 1978), will be modified and greatly extended to cover activation as well as inactivation phenomena.

Recently Moore, Hartig \& Raftery (1979) introduced the technique of controlled reduction of the number of activatable channels through binding of inhibitors. The expressions we present for the fractional distribution of inhibited channels over the microsac population, will permit quantitative determinations using this method.

\section{Single Microsac Flux}

In practice, initial conditions for flux experiments can be chosen to yield either a pure efflux of tracer (final number of tracer ions in a microsac is effectively zero), or a pure influx (initial number of tracer ions in a microsac is effectively zero). The respective flux events are describable as stochastic birth and death processes (see Appendix A). The time rate of change of the mean number of internal tracer ions $\bar{X}_{i}(t)$, of a single vesicle $i$ is

$$
\frac{\mathrm{d} \bar{X}_{i}}{\mathrm{~d} t}=-k n_{i} \alpha(t) \bar{X}_{i}(t)
$$


where here and throughout the following treatment the bars denote mean quantities. In equation (1) $k$ is the intrinsic rate constant for ion transport through a single channel, $n_{i}$ is the total number of activatable channels, and $\alpha(t)$ is the fraction of these that are open at time $t$. These equations are strictly valid only if tracer ion flux is not coupled to other non-equilibrium flux processes. However, experimental conditions can be adjusted to comply with this condition (Hess et al., 1975; Bernhardt \& Neumann, 1978).

The integrated solutions of equation (1) are for efflux:

$$
\bar{X}_{i}(t)=\bar{X}_{i}(0) \mathrm{e}^{-n_{i} \kappa(t)}
$$

where $\bar{X}_{i}(0)$ is the initial internal concentration of tracer, and for influx

$$
\bar{X}_{i}(t)=\vec{X}_{i}(0)\left[1-\mathrm{e}^{-n_{\mathrm{i}} \kappa(t)}\right]
$$

where $\bar{X}_{i}(0)$ is the initial external concentration of tracer. The amplitude factor

$$
\kappa(t)=k \int_{0}^{t} \alpha(t) \mathrm{d} \tau
$$

is necessary to account for the amount of tracer that has flown during the time intervals prior to $t$. Adoption of a time-dependent $\alpha(t)$ is indicated, to incorporate the time dependence of gating events that control the state of activation of channels.

A purely exponential flux is expected only if the gating process (a) results in a permanent activation of channels i.e. $\alpha=$ constant, (b) is rapid on the time scale of flux events. If gating and flux occur on the same time scale, both the flux time course, and the flux amplitude depend on the kinetics of gating.

\section{Overall Flux}

The total number of internal tracer ions, $X(t)$, is a sum of individual contributions, $x_{i}(t)$, due to all microsacs $i$

$$
X(t)=\sum_{i} x_{i}(t)
$$

For practical reasons it is difficult to prepare a perfectly homogeneous population of microsacs. One obtains instead, a mixture of vesicles, differing in size, and total number of activatable channels.

For the purpose of statistical averaging, the mixed population of a total of $\nu$ vesicles may be divided into subsets of $\nu_{n}$ vesicles, each having $n$ activatable channels per vesicle, where $n=0,1,2 \ldots, n_{\max }$ such that $\nu=\Sigma_{n} \nu_{n}$. Assuming the membrane fragments derive from tissue of fairly uniform 
channel density, it is plausible that the number of channels per microsac, and the microsac volume are strongly covariant. This implies that one can express the initial equilibrium number of tracer ions $x_{n}(0)$ of a given microsac in subset $n$ by a probability distribution $P_{n}$, such that

$$
\nu_{n} x_{n}(0)=\nu \bar{x}(0) P_{n} .
$$

A fundamental assumption in our previous scheme of analysis (Bernhardt \& Neumann, 1978) was that the number of open channels $m(t)$ of any individual microsac can be approximated by the expression

$$
m(t)=n \alpha(t),
$$

where $\alpha(t)$ is the fraction of active channels. The equation describing overall efflux is

$$
\bar{X}(t)=\nu \bar{X}(0) \sum_{n=0}^{n_{\max }} P_{n} \exp [-n \kappa(t)]
$$

where

$$
\kappa(t)=k \int_{0}^{t} \alpha(\tau) \mathrm{d} \tau
$$

Modelling $P_{n}$ by a normal distribution, i.e. $P_{n}=P(n) \mathrm{d} n=$ $\left(2 \sigma^{2}\right)^{-1 / 2} \exp \left[-(n-\bar{n})^{2} / 2 \pi \sigma^{2}\right] \mathrm{d} n$, and substituting integration over $n$ for the summation, one obtains

$$
\bar{X}(t)=\nu \bar{x}(0) \exp \left\{-\bar{n} \kappa(t)\left[1-\frac{\sigma^{2}}{2 \bar{n}} \kappa(t)\right]\right\} .
$$

An analogous derivation yields the corresponding equation for overall influx:

$$
\bar{X}(t)=\nu \bar{x}(0)\left(1-\exp \left\{-\bar{n} \kappa(t)\left[1-\frac{\sigma^{2}}{2 \bar{n}} \kappa(t)\right]\right\}\right)
$$

Equations (10) express all variable quantities in flux experiments in explicit mathematical form.

\section{Distribution of Fractionally Populated Microsacs}

The assumption that the number of open channels, $m$, per microsac can be represented by equation (7), requires closer examination. It applies only to the ideal case where the fraction of open channels on any given microsac equals the total fraction of open channels in the entire microsac population. 
Actually a distribution in the number of open channels per microsac is to be expected. For simplicity, the following treatment will be for the case where all microsacs have the same number of activatable channels, $n$, though the conclusions also apply to the more general case of a mixed population. As shown in Appendix B for a single-step gating process, one can calculate the fraction $\mu_{m}$ of microsacs having exactly $m$ open channels; $\mu_{m}$ is the probability of finding a microsac with $m$ open channels. The mean number and variance of open channels per microsac, respectively, are therefore

$$
\begin{aligned}
\bar{m}(t) & =\sum_{m=1}^{n} m \mu_{m}(t) \\
\sigma^{2}(t) & =\overline{m^{2}}(t)-\overline{m(t)^{2}}
\end{aligned}
$$

where

$$
\overline{m^{2}(t)}=\sum_{m=1}^{n} m^{2} \mu_{m}(t)
$$

Assumption (7) is valid only if the ideal mean number of open channels $\bar{m}_{i}$ is equal to $\bar{m}$ calculated by cquation (11), and the probability distribution $\mu_{m}$ is sharply peaked [i.e. $\sigma^{2}(t)$ is small]. For a single-step gating process, with apparent forward and reverse rate constants $k_{1}^{\prime}$ and $k_{-1}^{\prime}$, respectively, one has

$$
\bar{m}_{i}(t)=\bar{m}_{i}(\infty)+\left[\bar{m}_{i}(0)-\bar{m}_{i}(\infty)\right] \exp \left[-\left(k_{1}^{\prime}+k_{-1}^{\prime}\right) t\right] .
$$

In order to estimate the validity of equation (7), calculations for a single-step channel opening process were performed:

(1) Computer solution of the matrix eigenvalue problem outlined in Appendix B was carried out. Eigensolutions $\mu_{m}(t)$, with $m=1, \ldots, n$, were determined for the initial condition $\mu_{m}(0)=0$ for $m=0$, and $\mu_{0}(0)=1$ (i.e. initially none of the channels are open).

(2) The calculations were performed for variable $n$, and $K^{\prime}$ where $K^{\prime}=$ $k_{1}^{\prime} / k_{-1}^{\prime}$ is the distribution constant for gating; see Appendix B. In order to limit the number of variables, time was scaled to units $1 / k_{-1}^{\prime}$ (i.e. $k_{-1}^{\prime}=1$ ).

(3) The ideal mean number of channels was calculated from equation (13) with $\bar{m}_{i}(0)=0$, and $\bar{m}_{i}(\infty)=n K^{\prime} / 1+K^{\prime}$.

Calculations were performed for $n=5,10,15,20$ and $K^{\prime}=1,10$ and 100 . In agreement with equation (7) it was found in all cases, that $m_{i}(t)=\bar{m}(t)$, where $\bar{m}(t)$ was calculated using equation (11), and that the maximum variance throughout the gating reaction, was always of the order of $n / 5$. Figure 1 shows the time development of the probability distribution $\mu_{m}(t)$, 


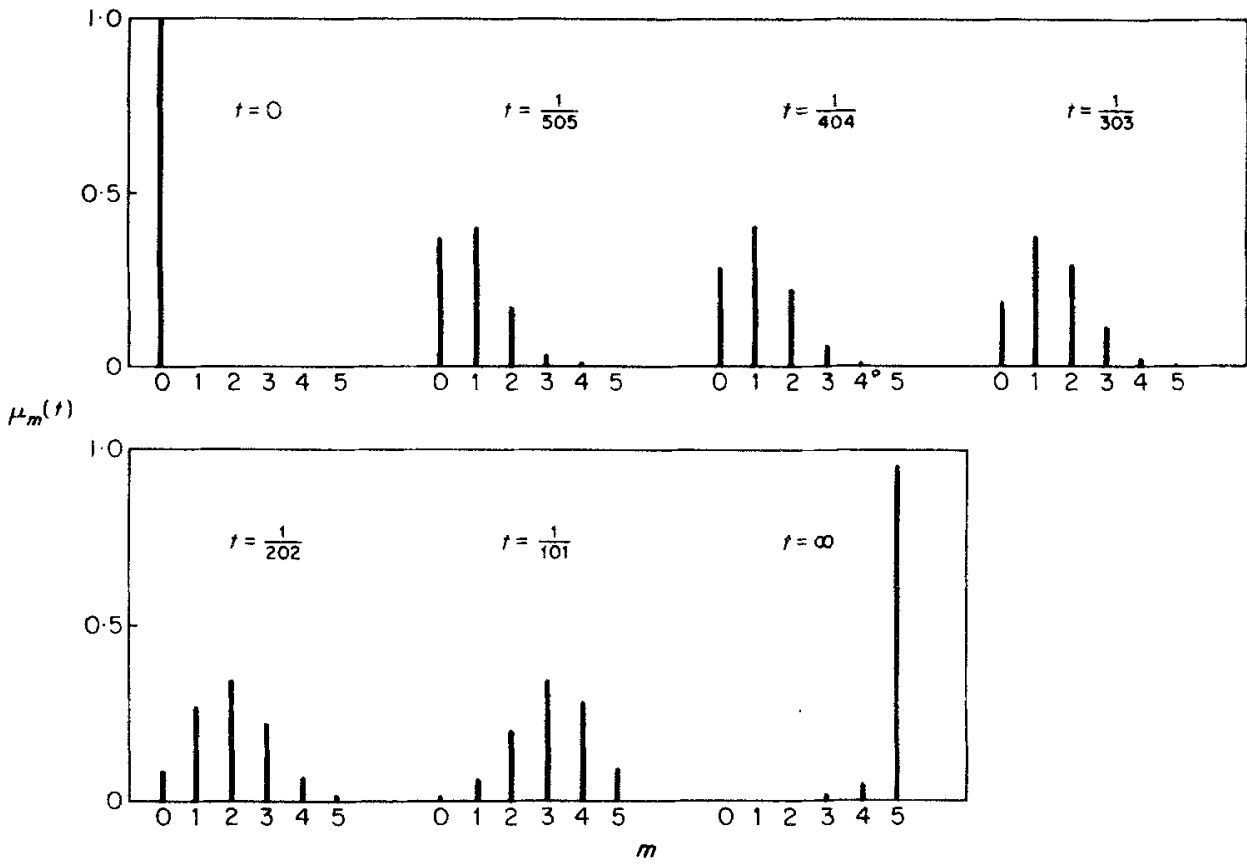

FIG. 1. The course of repopulation of microsacs according to reaction scheme (B1), for a one-step channel opening process, with $n=5, K^{\prime}=100$, at progressive times in units of $1 / k_{-1}^{\prime}$ : $t=0, t=1 \tau, 2 \tau \ldots 5 \tau$ and infinity (final equilibrium): see equation (B2).

for the representative case $n=5, K^{\prime}=100$. Table 1 summarizes the corresponding values of $\bar{m}(t), \bar{m}_{i}(t)$, and $\sigma^{2}(t)$.

These results indicate that the assumption of equation (7) is valid, and subsequently, that expressions (10) are correct.

The same formalism is equally applicable to a reversible one-step inactivation process. For this case, $\mu_{m}$ must be taken to represent the fraction of microsacs with $m$ inactivated channels.

\section{Irreversible Ligand Binding}

Equations (10) indicate that the total number of activatable channels per microsac $(n)$ is a fundamental variable in flux experiments. For many gating systems (e.g. acetylcholine receptors, axonal sodium channels) practically irreversibly acting, inhibitory ligands of high specificity exist. Systematic inhibition of the gating molecules, allows controlled reduction of the 


\section{TABLE 1}

The ideal mean number of open channels $\left(\bar{m}_{i}\right)$, the actual mean $(\bar{m})$, and the variance $\left(\sigma^{2}\right)$ for a one-step channel opening process, with $n=5, K^{\prime}=100$ at progressive times in units of $1 / k_{-1}^{\prime}: t=0, t=1 \tau, 2 \tau, \ldots 5 \tau$, and infinity

( $f$ inal equilibrium); see equation $(B 2)$

\begin{tabular}{llll}
\hline Time & \multicolumn{1}{r}{$\bar{m}_{i}$} & \multicolumn{1}{c}{$\bar{m}$} & \multicolumn{1}{c}{$\sigma^{2}$} \\
\hline 0.000 & 0 & 0 & 0 \\
$1 / 505$ & 0.897 & 0.897 & 0.736 \\
$1 / 404$ & 1.095 & 1.095 & 0.855 \\
$1 / 303$ & 1.403 & 1.403 & 1.00947 \\
$1 / 202$ & 1.948 & 1.948 & 1.18903 \\
$1 / 101$ & 3.129 & 3.129 & 1.171 \\
$\infty$ & 4.950 & 4.950 & 0 \\
\hline
\end{tabular}

number of activatable channels; $n$ is therefore an experimentally accessible variable quantity in flux experiments.

Often, the stoichiometry of the inhibition reaction, and the total number $N$ of gating molecules in the preparation are known. The overall ratio $\alpha=M / N$, of irreversibly inactivated to total gating molecules can therefore be adjusted by application of a determinate amount of the inhibitor. The constraints that $M$ of $N$ channels are permanently inactivated leads to a statistical distribution of the number of activatible channels per microsac. One can determine the fraction $\mu_{m}$, of microsacs that have exactly $m$ inactivated channels. As shown in Appendix $C$,

$$
\mu_{m}=\left(\begin{array}{c}
n \\
m
\end{array}\right) \alpha^{m}(1-\alpha)^{n-m} .
$$

Two useful applications of controlled, irreversible inhibition of gating molecules exist:

(1) One can "titrate" with inhibitor, and determine $\bar{n}$ and $\sigma^{2}$ at a fixed time from equation (10)-all other factors being held equal.

(2) One can use the relationship resulting from equation (14) for $m=n$, i.e. $\mu_{n}=\alpha^{n}$. Averaging over $P_{n}$ leads to the mean fraction having all channels inhibited:

$$
\bar{\mu}_{n}=\alpha^{\bar{n}\left[1-\sigma^{2} / 2 \bar{n} \ln \alpha\right]} .
$$

The quantity $\bar{\mu}_{n}$ can be directly determined from the flux amplitude $P$, since it is proportional to the decrease in amplitude $\Delta P$ due to reduction of 
microsacs contributing to flux, i.e. $\bar{\mu}_{n}=\Delta P / P$. Again, titration with inhibitor, all other factors being held equal, permits determination of $\bar{n}$ and $\sigma^{2}$, according to the linear relationship $\ln \bar{\mu}_{n} / \ln \alpha=\bar{n}-\frac{1}{2} \sigma^{2} \ln \alpha$.

\section{Discussion}

Investigation of the flux of ions from or into sealed membrane fragments, cells, or vesicles, is a generally applicable method for studying the function of gating molecules. The native environment of the gating system can be preseryed as much as is possible in an in vitro measurement, yet a multitude of factors influencing the gating process are accessible to accurate experimental control. We have shown that the overall flux signal is explicitly dependent on a number of physico-chemically well-defined variables. The fundamental expression (10) for the total number of tracer ions $\bar{X}(t)$, can be recast to illustrate the connection with measurable quantities

$$
\bar{X}(t)=D V C_{0} \bar{v} \exp \left\{-\bar{n} \kappa(t)\left[1-\frac{\sigma^{2}}{2 \bar{n}} \kappa(t)\right]\right\},
$$

where $D=$ density of the suspension (microsacs/unit volume), $V=$ volume of suspension, $C_{0}=$ initial concentration of tracer ions, $\bar{v}=$ average volume of a microsac, $\bar{n}=$ mean total number of receptor controlled channels per microsac, $\sigma^{2}=$ variance in number of receptor controlled channels per microsac, $\kappa(t)=$ amplitude factor.

Aside from $\kappa(t)$, all variables are functions of the method of preparation, and of the materials used. They can be determined prior to the flux experiment-thus correcting for all sources of variability between preparations.

All information about the gating process is contained in $\kappa(t)$, given by equation (9). As shown previously (Bernhardt \& Neumann, 1978) for ligand induced inactivation of the acetylcholine receptor, the time-dependent fraction of open channels $\alpha(t)$, can be determined from the time course of the flux signal.

An extremely useful fact to emerge from our analysis, which has recently also been utilized by Moore, Hartig \& Raftery (1979), is that flux amplitudes themselves can contain information about the gating reaction. The frequently encountered case of a rapid activation phase followed by slow inactivation can be used as an illustration. The number of open channels prior to activation, and following completion of inactivation, is assumed to be negligibly small. The time dependent fraction of open channels is then

$$
\alpha(t)=\alpha_{0} \mathrm{e}^{-k_{i} t}\left(1-\mathrm{e}^{-k_{a} t}\right),
$$




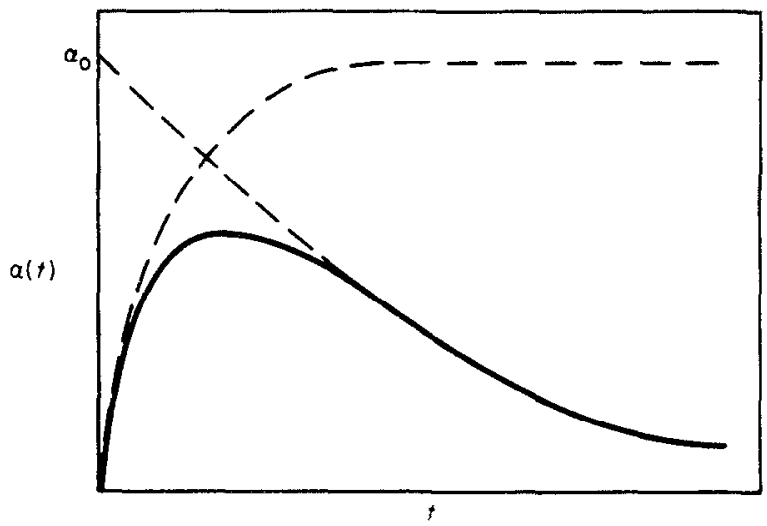

FIG. 2. The fraction of open channels $\alpha(t)$ for rapid activation of the gating system, followed by slow inactivation [equation (17)] as a function of time, $t ; \alpha_{0}$ is the amplitude of both the activation, and the inactivation reaction.

where $\alpha_{0}$ is the amplitude for activation and inactivation (see Fig. 2). $k_{a}$ and $k_{i}$ are, respectively reciprocal time constants for activation and inactivation. The corresponding amplitude factor [equation (9)] is

$$
\kappa(t)=\alpha_{0} k\left[\frac{\mathrm{e}^{-\left(k_{a}+k_{i}\right) t}-1}{k_{a}+k_{i}}-\frac{\mathrm{e}^{-k_{i} t}-1}{k_{i}}\right] .
$$

For practical reasons it may not be possible to resolve the time course of the rapid activation phase. Knowledge of the initial time course of inactivation, assuming essential completion of activation, suffices to determine $k_{a}$. In that time range one has $\mathrm{e}^{-k_{a} t} \ll 1, \mathrm{e}^{-k_{i} t} \cong 1-k_{i} t$, and $k_{a} \gg k_{i}$, which yields, upon substitution into equation (18),

$$
\kappa(t) \cong \alpha_{0} k\left(t-\frac{1}{k_{a}}\right) .
$$

Slope and intercept of this linear function of time allow determination of $\alpha_{0} k$ and $k_{a}$. The overall amplitude factor $\kappa(\infty)=\alpha_{0} k / k_{i}$, then permits further determination of $k_{i} . k_{a}$ and $k_{i}$ are themselves functions of the elementary rate constants for the gating reaction, and of external factors that influence gating (e.g. activator ligand concentration, membrane potential, ions in the external medium).

In summary, it has been shown that detailed analysis of tracer flux data can yield quantitative information about the kinetics of gating processes. Inhomogeneities in the preparation, and other sources of deviations from 
ideal behaviour, have been explicitely considered. The only serious limitation to a general application is, in practice, the requirement that the flux process must be at least as rapid as the gating process.

We gratefully acknowledge financial support by the Deutsche Forschungsgemeinschaft, grant $\mathrm{Ne} 227$.

\section{REFERENCES}

Bernhardt, J. \& Neumann, E. (1978). Proc. natn. Acad. Sci. U.S.A. 75, 3756.

EIGEN, M. \& DEMAYER, L. (1973). Techniques of Chemistry, vol. 6, (A. Weissberger \& G. G. Hammes eds), p. 63. New York: Wiley.

Hess, G., Andrews, J. P., Stuve, G. F. \& Coombs, S. E. (1975). Proc. natn. Acad. Sci. U.S.A. 72, 4371.

Hess, G. P., DeREK, J. C. \& AOSHIMA, H. (1979). Nature 282, 329.

ILGENFRITZ, G. (1977). Chemical Relaxation in Molecular Biology (I. Pecht \& R. Rigler eds), p. 1. New York: Springer.

KASAI, M. \& ChangeuX, J.-P. (1971). J. Memhr. Biol. 6, 1.

LANDIS, C. A. \& PUTNey JR., J. W. (1979). J. Physiol. 297, 369.

MCQuARrie, D. A. (1975). Physical Chemistry an Advanced Treatise, vol. 9, (Henderson ed.), Ch. 10. New York: Academic Press.

Miller, D., MOORE, H.-P., HARTig, P. \& Raftery, M. A. (1978). Biochem. biophys. Res. Commun. 85, 632.

MoOre, H.-P., HARTig, P. R. \& RAFTery, M. A. (1979). Proc. natn. Acad. Sci. U.S.A. 76, 6265 .

Popot, J. L., SugiYama, H. \& ChangeuX, J.-P. (1976). J. mol. Biol. 106, 469.

Villegas, R., Villegas, G. M., Barnola, F. V. \& RACKer, E. (1977). Biochem. biophys. Res. Commun. 79, 210.

Villegas, R., Villegas, G. M., Barnola, F. V. \& Racker, E. (1979). Adv. Cytopharmacol. 3, 373 .

YANNICK, J., FosSET, M. \& LAZDUNSKI, M. (1978). J. biol. Chem. 253, 7383.

\section{APPENDIX A}

Under suitable conditions, efflux of substance from a single microsac can be modelled as a stochastic process discrete in sample space but continuous in time-a pure death process (McQuarrie, 1975). The probability $P_{x}(t)$, of finding $x$ molecules still inside the microsac at time $t$, obeys the differential difference equation

$$
\mathrm{d} P_{x} / \mathrm{d} t=-r x P_{x}(t)+r(x+1) P_{x+1}(t)
$$

where $r$ is the rate constant for efflux from the microsac. The solution of this equation is

$$
\begin{aligned}
P_{x}(t) & =\left(\begin{array}{c}
x_{0} \\
x
\end{array}\right) \exp \left(-x_{0} r t\right)\left(\mathrm{e}^{r t}-1\right)^{x_{0}-x} & & \text { for } 0 \leq x \leq x_{0} \\
& =0 & & \text { for } x>x_{0},
\end{aligned}
$$


where $x_{0}$ is the number of molecules in the microsac at $t=0$. The mean number of internal molecules at a given time is

$$
\bar{x}(t)=\sum_{x=0}^{\infty} x P_{x}(t)=x_{0} \mathrm{e}^{-r t} .
$$

This is precisely the result obtained from the conventional deterministic treatment (i.e. the solution of $\dot{\bar{X}}=-r \bar{X}$ ). The variance is

$$
\sigma^{2}(t)=\overline{x^{2}}-\bar{x}^{2}=x_{0} \mathrm{e}^{-r t}\left(1-\mathrm{e}^{-r}\right) .
$$

An entirely analogous result may be obtained for influx of molecules.

\section{APPENDIX B}

The reaction scheme for a process involving microsacs with $n$ gating units, for which each gating unit undergoes a reversible change is

$$
N_{0} \underset{k_{-1}^{\prime}}{\stackrel{n k_{1}^{\prime}}{\rightleftharpoons}} N_{1} \underset{2 k_{-1}^{\prime}}{\stackrel{(n-1) k_{1}^{\prime}}{\rightleftharpoons}} N_{2} \ldots N_{n-1} \underset{n k_{-1}^{\prime}}{\stackrel{k_{1}^{\prime}}{\rightleftharpoons}} N_{n},
$$

where $N_{m}$ represents microsacs on which $m$ gating units have reacted. During the course of the reaction a repopulation among species $N_{m}$ takes place. The fraction $\mu_{m}$, of microsacs in form $N_{m}$, can be determined from the kinetic equations for the reaction scheme. In equation (B1), the intrinsic reaction step may involve the binding of an activator $\mathrm{A}$ to the gating system $\mathrm{G}$ according to $A+N_{r-1} \rightleftharpoons N_{r}$ with the intrinsic rate constants $k_{1}$ and $k_{-1}$. When the concentration of activator is large compared to the number of binding sites, $k_{1}^{\prime}=k_{1}[A], k_{-1}^{\prime}=k_{-1}$, and $K^{\prime}=K[A]$ where $K=k_{1} / k_{-1}$ is the intrinsic equilibrium constant. Therefore when experimental conditions can be adjusted so that all second-order steps become pseudo first order steps (e.g. buffer conditions for ligand binding), one can find explicit solutions for eigenvalues (inverse rate constants), and eigenvectors (normal modes) (Eigen \& DeMayer, 1973; Ilgenfritz, 1977). The time dependent fraction $\mu_{m}$ is

$$
\begin{aligned}
& \mu_{m}(t)=\mu_{m}(\infty)+\sum_{r=1}^{n} w_{m r} \mathrm{e}^{-t / \tau_{r}} \sum_{l=1}^{n} \tilde{w}_{r l}\left[\mu_{l}(0)-\mu_{l}(\infty)\right], m>0 \\
& \mu_{0}(t)=1-\sum_{r=1}^{n} \mu_{r}(t) .
\end{aligned}
$$

The reciprocal time constants are

$$
\frac{1}{\tau_{r}}=r\left(k_{1}^{\prime}+k_{-1}^{\prime}\right) \quad r=1, \ldots, n .
$$


A similarity transformation by the matrix $\mathbf{W}$ (elements $w_{i j}$ ) and its inverse $\mathbf{W}^{-1}$ (elements $\bar{w}_{i j}$ ) diagonalizes the matrix of kinetic coefficients; here

$$
w_{m, r+1}=\frac{(-1)^{r}}{r !} \sum_{l=\sigma}^{\zeta}\left(\begin{array}{l}
r \\
l
\end{array}\right) \frac{(n-m) !}{(n-m-l) !} \frac{m !}{(m+l-r) !}\left(-K^{\prime}\right)^{l}
$$

and $w_{m 0}=1$.

$K^{\prime}$ is the distribution constant $K^{\prime}=k_{1}^{\prime} / k_{-1}^{\prime}$, and

$$
\sigma=\left\{\begin{array}{l}
0 \\
m-1
\end{array} \text { for } \begin{array}{l}
r<m \\
r \geqslant m
\end{array} \quad \zeta=\left\{\begin{array}{l}
r \\
n-m-1
\end{array} \text { for } \begin{array}{l}
r<n-m \\
r \geq n-m .
\end{array}\right.\right.
$$

The equilibrium fractions are given by

$$
\mu_{m}(\infty)=\left(\begin{array}{c}
n \\
m
\end{array}\right) K^{\prime m}\left(1+K^{\prime}\right)^{-n}
$$

\section{APPENDIX C}

The following analysis is applicable to all problems involving the random selection of $M$ of a total of $N$ elements from a set of $\nu$ units, each bearing $n$ elements, so that $N=n \nu$. There are $\left(\begin{array}{l}N \\ M\end{array}\right)$ ways of selecting the $M$ elements. This corresponds to all possible configurations that arise from dividing $M$ into subsets $\left\{m_{i}\right\}$, such that $m_{i}<n$ elements are selected from the $i$ th unit, and $\Sigma_{i} m_{i}=M$; therefore

$$
\left(\begin{array}{c}
N \\
M
\end{array}\right)=\sum_{\left\{m_{i}\right\}} \prod_{j}\left(\begin{array}{c}
n \\
m_{j}
\end{array}\right)
$$

The largest individual contribution to the sum arises from the configuration where each $m_{i}=\bar{m}$. However, since all configurations having $\nu_{m}$ microsacs with $m_{i}=m$ are indistinguishable from an experimental point of view, there will be $\nu ! / \Pi_{m} \nu_{m}$ ! ways of choosing a given arrangement $\left\{\nu_{m}\right\}$. The most probable distribution $\left\{\bar{\nu}_{m}\right\}$ can be found by determining the largest contribution to the sum

$$
\nu \sum_{\left\{\nu_{m}\right.} \prod_{m} \frac{1}{\nu_{m} !}\left(\begin{array}{c}
n \\
m
\end{array}\right)^{\nu_{m}}
$$

under the constraints $\Sigma_{m} \nu_{m}=\nu$, and $\Sigma_{m} m \nu_{m}=M$.

Straightforward application of the method of Lagrangian multipliers, leads to an expression for the fraction $\bar{\mu}_{m}$ of the most probable distribution

$$
\bar{\mu}_{m} \equiv \frac{\bar{\nu}_{m}}{\nu}=\left(\begin{array}{c}
n \\
m
\end{array}\right) \alpha^{m}(1-\alpha)^{n-m}
$$

where $\alpha$ is the probability of a selection. 


\section{Addendum}

The fundamental equations (10a) and (10b) are readily modified to cover the reduction of the total fraction $\alpha$ of receptors contributing to flux, following irreversible inhibition. Inserting equations (14) and (8) into equations $(10 a, b)$ yields for efflux:

$$
\begin{aligned}
\bar{X}(\alpha, t) & =\nu \bar{x}(0) \sum_{n=0}^{n_{\max }} P_{n} \sum_{m=0}^{n} \mu_{m} \exp \left[-(n-m)_{\kappa}(t)\right] \\
& =\nu \bar{x}(0) \int P(n)[\lambda(\alpha, t)]^{n} \mathrm{~d} n \\
& =\nu \bar{x}(0)[\lambda(\alpha, t)]^{\bar{n}-\sigma^{2} / 2 \ln \lambda(\alpha, t)}
\end{aligned}
$$

where

$$
\lambda(\alpha, t)=\mathrm{e}^{-\kappa(t)}+\alpha\left[1-\mathrm{e}^{-\kappa(t)}\right],
$$

and for influx:

$$
\bar{X}(\alpha, t)=\nu \bar{x}(0)\left\{1-[\lambda(\alpha, t)]^{\bar{n}-\sigma^{2} / 2 \ln \lambda(\alpha, t)}\right\} .
$$

Dependence of $\bar{X}(\alpha, \infty)$ on $\alpha$ can be utilized to determine $\bar{n}$ and $\sigma^{2}$ experimentally (Bernhardt \& Neumann, 1980, Neurochem. Int., in press). Furthermore, the proof that $\bar{m}(t)=\bar{m}_{i}(t)$, see equations (11) and (13) is simple. For perfectly random ligand binding to equivalent sites,

$$
\mu_{m}(t)=\left(\begin{array}{c}
n \\
m
\end{array}\right)[\alpha(t)]^{m}[1-\alpha(t)]^{n-m}
$$

where the time-dependent fraction $\alpha(t)$ of ligand-occupied sites is given by $\alpha(t)=\alpha(\infty)+[\alpha(0)-\alpha(\infty)] \exp \left[-\left(k_{1}^{\prime}+k_{-1}^{\prime}\right) t\right]$. This expression for $\mu_{m}(t)$ is identical to the expression derived in Appendix B. Thus the distribution remains binomial throughout the repopulation (B1); therefore equation (11) gives:

$$
\bar{m}(t)=\sum_{m=0}^{n} \mu_{m}(t)=n \alpha(t)=\bar{m}_{i}(t)
$$

and equation (12) is

$$
\sigma^{2}(t)=n \alpha(t)[1-\alpha(t)]
$$

For the case of ligand-induced activation discussed in the text $\alpha(0)=0$ and $\alpha(\infty)=K^{\prime} /\left(1+K^{\prime}\right)$. 